\title{
Pre-Treatment and Post-Treatment Neutrophil-to- Lymphocyte Ratio Predict Pathological Tumor Response and Survival in Rectal Cancer Patients Treated with Neoadjuvant Chemoradiotherapy
}

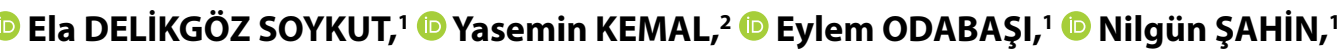 \\ (1) Betül KESKINKILIÇ YAĞIZ, ${ }^{3}$ (D) İrem Melike YAZICIOĞLU, ${ }^{4}$ (i) Süheyla AYTAÇ ARSLAN, ${ }^{5}$ (i) YıIdız GÜNEY5
}

\author{
'Department of Radiation Oncology, University of Health Sciences, Samsun Training and Research Hospital, Samsun-Turkey \\ ${ }^{2}$ Department of Medical Oncology, Istinye University, Istanbul-Turkey \\ ${ }^{3}$ Department of General Surgery, Gazi State Hospital, Samsun-Turkey \\ ${ }^{4}$ Department of Pathology, University of Health Sciences, Samsun Training and Research Hospital, Samsun-Turkey \\ ${ }^{5}$ Department of Radiation Oncology, Memorial Hospital, Ankara-Turkey
}

\begin{abstract}
OBJECTIVE
The aim of this study is to investigate the relationship between baseline and post-treatment neutrophilto-lymphocyte ratio (NLR) levels and response to neoadjuvant chemoradiotherapy (CRT) in terms of good pathological response and survival.
\end{abstract}

\section{METHODS}

Fifty-six patients who underwent neoadjuvant CRT and curative surgery for locally advanced rectal cancer (LARC) were analyzed retrospectively. Pre-CRT and post-CRT hematologic parameters were recorded. The link between NLR and clinical outcomes was explored.

\section{RESULTS}

The receiver operating characteristic analysis revealed appropriate cut-off values of 2.87 for pre-CRT NLR associated with good pathological response and 8.68 for post-CRT NLR predicting survival. The low pre-CRT NLR group had better outcomes in terms of good pathological response compared to the high pre-CRT NLR group (OR 4.15, 95\% CI 1.23-13.76, $\mathrm{p}=0.021$ ). However, the analysis failed to show the correlation between NLR and pCR (OR 2.74, 95\% CI 0.37-20.15, $\mathrm{p}=0.320$ ). Patients with elevated post-CRT NLR had significantly worse 5 -year overall survival (OS), disease-free survival (DFS) and local regional recurrence-free survival (LRRFS) rates compared to low post-CRT NLR in multivariate analysis ( $46.6 \%$ vs. $74.4 \%, \mathrm{p}=0.020 ; 35.3 \%$ vs. $71.9 \%, \mathrm{p}=0.018 ; 40.8 \%$ vs. $78.1 \%, \mathrm{p}=0.006$ ).

\section{CONCLUSION}

High pre-CRT NLR might be used as a poor pathological tumor response predictor in LARC patients treated with neoadjuvant CRT. In addition, low post-CRT NLR is associated with favorable OS, DFS, and LRRFS. Therefore, easily accessible and cost-effective NLR can be considered as a potential predictive marker to identify patients and establish personalized treatment strategies.

Keywords: Neoadjuvant chemoradiotherapy; neutrophil-to-lymphocyte ratio; pathological tumor response; prognosis; rectal cancer; survival.

Copyright $\odot$ 2022, Turkish Society for Radiation Oncology

Dr. Ela DELIKGÖZ SOYKUT

Sağlık Bilimleri Üniversitesi,

Samsun Eğitim ve Araştırma Hastanesi,

Radyasyon Onkolojisi Bölümü,

Samsun-Turkey

E-mail: eladelikgoz@gmail.com 


\section{Introduction}

Neoadjuvant chemoradiotherapy (CRT) followed by curative surgery including total mesorectal excision (TME) and adjuvant chemotherapy has been the current standard treatment option for patients with locally advanced rectal cancer (LARC). In the early 2000s, when the German Rectal Cancer Study Group's phase three randomized clinical trial was published, it was recognized worldwide for its superior results, favoring neoadjuvant CRT over postoperative CRT because of better sphincter preservation and improved local control, as well as reduced treatment-related toxicity.[1] While downstaging is provided in approximately 50$60 \%$ of patients with neoadjuvant CRT, pathological complete response (pCR) is observed at a rate of $10 \%$. $[1,2]$ Patients who achieve pCR after neoadjuvant CRT have better survival and $\mathrm{PCR}$ has been proposed as a reliable prognostic factor for survival in LARC patients. [2] Since pCR is important in determining prognosis, identifying factors affecting $\mathrm{PCR}$ prior to neoadjuvant CRT may be strategically important for deciding patient management. In this situation, it is still necessary to identify specific biomarkers to predict the treatment response of patients undergoing neoadjuvant CRT.

Accumulating evidence has supported that systemic inflammation has been recognized as a hallmark of cancer development and progression. The systemic inflammatory response plays a crucial role in the prognosis of several solid tumors due to interactions of the tumor microenvironment resulting in tumor growth and spread. [3] Neutrophils, lymphocytes, and platelets are immuneinflammatory circulating cells derived from peripheral blood. The neutrophil-to-lymphocyte ratio (NLR) is one of the immune-inflammatory indices that have been shown to be associated with the prognosis for the survival of various types of solid tumors.[4,5] Although the exact mechanism is not fully understood, it can be briefly summarized as follows. Neutrophils secrete cytokines and chemokines, that create a favorable tumor microenvironment to promote tumor growth and progression; lymphocytes are responsible for the antitumor immune status due to their role in destroying tumor cells, suppressing tumor proliferation and migration. Therefore, increased NLR is assumed to be associated with tumor growth.

In this study, our primary goal is to investigate the relationship between baseline and post-treatment NLR levels and response to neoadjuvant CRT in terms of pCR and good pathological response. We also aimed to evaluate the prognostic ability of pre-CRT and postCRT NLR on survival outcomes.

\section{Materials and Methods}

\section{Patients}

LARC patients treated with neoadjuvant CRT in our radiation oncology department between January 2012 and December 2017 were retrospectively reviewed. The following eligibility criteria were considered in the present study: pathologically confirmed diagnosis of rectum adenocarcinoma; clinical T3/T4 or lymph node-positive disease; including treatment with neoadjuvant CRT followed by curative surgery; blood tests were taken within 1 week before CRT (pre-CRT NLR) and between completion of CRT and surgery (postCRT NLR), and an Eastern Cooperative Oncology Group performance score of 0-2. Patients who used steroid therapy had signs of inflammation or infectious disease, had a history of hematological or autoimmune disease, and had other tumors were excluded from the study. The study was approved by the medical ethics committee of our institute and was conducted in accordance with the Declaration of Helsinki, and individual consent was waived for this retrospective study.

\section{Data Collection}

Patient demographics, surgical and oncologic outcomes were extracted through the patient archive files and electronic medical records system, including age, gender, tumor location, carcinoembryonic antigen (CEA), albumin, uric acid, hemoglobin, neutrophil, lymphocyte, monocyte and platelet counts, clinical $\mathrm{T}$ and $\mathrm{N}$ stage, type of surgery, lymphovascular invasion (LVI), perineural invasion (PNI), pathological TNM stage, recurrence, distant metastasis, and death. The NLR was calculated as the neutrophil count divided by the lymphocyte count and was categorized as pre-CRT and post-CRT.

\section{Pretreatment Assessment}

The clinical workup included physical examination, blood analysis, colonoscopy, computed tomography scan (CT) of the abdomen, pelvis and chest, and pelvic magnetic resonance imaging for pretreatment evaluation. In addition, positron emission tomography was performed on several patients. The seventh edition of the TNM classification of the American Joint Committee on Cancer was used to define the clinical stage.

\section{Treatment}

Three-dimensional radiotherapy was applied with a median radiation dose of $50.4 \mathrm{~Gy}$. Radiotherapy was performed to the whole pelvis with $45 \mathrm{~Gy}$ in 25 fractions, followed by a boost of 5.4 Gy to the primary tumor in 
3 fractions. The gross tumor volume (GTV) contained primary tumor and involved lymph nodes visible on imaging. The clinical target volume (CTV) was defined including the entire mesorectum and associated lymph nodes. The lymph nodes consisted of obturator and internal iliac lymph nodes for all $\mathrm{T}$ stages and external iliac lymph nodes for T4 tumors. The CTV was enlarged $1-1.5 \mathrm{~cm}$ in adjacent organs with gross tumor invasion for T4 tumors. The boost volume covered the GTV with a $2 \mathrm{~cm}$ margin superiorly-inferiorly and at these levels with the mesorectum and presacral space. The CTV was expanded 5-10 $\mathrm{mm}$ to create the planning target volume. $\mathrm{kV}$ imaging was done daily or every other day for set-up accuracy. The treatment was applied with a full bladder to minimize small bowel toxicity.

Concomitant chemotherapy was administered, except for 4 patients who could not receive chemotherapy due to their age and comorbidities during radiotherapy. Intravenous 5-fluorouracil (5-FU) or oral capecitabine chemotherapy regimens were used. In the $1^{\text {st }}$ and $5^{\text {th }}$ weeks of RT, $425 \mathrm{mg} / \mathrm{m}^{2} /$ day 5 -FU and $20 \mathrm{mg} / \mathrm{m}^{2} /$ day leucovorin were given. Capecitabine was administered at $825 \mathrm{mg} / \mathrm{m}^{2} /$ twice daily during the entire radiotherapy period. All patients underwent curative surgery including TME and pelvic node dissection 4-12 weeks after the completion of neoadjuvant CRT, and adjuvant chemotherapy was applied.

\section{Pathological Assessment of Response to Che- moradiation}

As described by previous reports by Ryan et al.[6] the pathologic response to neoadjuvant CRT was determined by the amount of viable malignancy versus the amount of fibrosis and was defined in 5 tumor regression grades (TRG). In brief, TRG1 was defined as no viable cancer cells (complete response); TRG2 as remaining of single cells or rare small groups of cancer cells (near-complete response); TRG3 as the presence of residual cancer with predominantly fibrosis (partial response); TRG4 as residual cancer outgrowing fibrosis; TRG5 as extensive residual cancer without fibrosis. The pCR was defined as the absence of all malignant cells, a good pathological response to neoadjuvant CRT was defined as TRG1-3, while a poor pathological response was defined as TRG4-5.

\section{Follow-up}

Patients were followed up every 3 months within the first 2 years, every 6 months in the following 3 years, and annually thereafter. Physical examinations, complete blood count, blood chemistry, CEA were performed at each follow-up. Patients were evaluated with chest and abdominal CT scan at 6-month intervals. Total colonoscopy was performed at 1 year after surgery and every 2 years thereafter. Based on clinical, radiologic evaluations, or histological findings, any recurrence in the pelvic cavity was defined as locoregional recurrence, any recurrence outside the pelvic cavity, or metastasis in solid organs, was defined as distant metastasis.

\section{Statistical Analysis}

The primary endpoints were $\mathrm{pCR}$ and good pathological response to neoadjuvant CRT by TRG stages as previously described. The secondary clinical outcomes of interest were overall survival (OS), disease free survival (DFS), and local-regional recurrence-free survival (LRRFS). OS was defined as the time from the date of diagnosis to the date of death or last followup. The time from the date of diagnosis to the date of occurence of local or/and distant failure, or death, or last follow-up was calculated as DFS; the time from the date of diagnosis to the local recurrence, or death, or last follow-up was calculated as LRRFS.

The statistical analyses were performed with IBM SPSS version 21.0 (IBM Corp., Armonk, NY, USA). A $\mathrm{p}<0.05$ was considered significant for all tests. Descriptive analysis was used for all variables. Categorical variables were displayed as frequencies and percentages, continuous variables were represented as medians. The receiver operating characteristic (ROC) analysis was applied to determine the optimal cut-off values of preCRT NLR and post-CRT NLR for prognostic prediction of good pathological response to neoadjuvant CRT and survival. The relationship between the NLR and the clinicopathological characteristics were compared using the chi-square test or Fisher's exact test for categorical variables and with Student's t-test for continuous variables. Logistic regression analysis was used to analyze the significant factors related to response to neoadjuvant CRT. The patients' survival data were evaluated using the KaplanMeier method and the differences were compared using log-rank statistics. Univariate and multivariate analyses to identify prognostic predictors were performed using Cox proportional hazard regression models.

\section{Results}

The demographic, clinical, and pathological characteristics of this study are displayed in Table 1. Of the 110 patients enrolled at baseline, 56 met eligibility criteria 


\begin{tabular}{|c|c|c|c|c|c|c|}
\hline \multirow[t]{2}{*}{ Variable } & \multicolumn{2}{|c|}{ Pre-CRT NLR (\%) } & \multirow[t]{2}{*}{$\mathbf{p}$} & \multicolumn{2}{|c|}{ Post-CRT NLR (\%) } & \multirow[t]{2}{*}{$\mathbf{p}$} \\
\hline & $<2.87$ & $\geq 2.87$ & & $<8.68$ & $\geq 8.68$ & \\
\hline \multicolumn{7}{|l|}{ Age } \\
\hline$<60$ & $8(34.8)$ & $13(39.4)$ & 0.726 & $11(34.4)$ & $8(38.1)$ & 0.782 \\
\hline$\geq 60$ & $15(65.2)$ & $20(60.6)$ & & $21(65.6)$ & $13(61.9)$ & \\
\hline \multicolumn{7}{|l|}{ Gender } \\
\hline Female & $10(43.5)$ & $15(45.5)$ & 0.884 & $14(43.8)$ & $10(47.6)$ & 0.782 \\
\hline Male & $13(56.5)$ & $18(54.5)$ & & $18(56.2)$ & $11(52.4)$ & \\
\hline \multicolumn{7}{|l|}{ Tumor location } \\
\hline Low-Mid & $16(72.7)$ & $26(81.3)$ & 0.459 & $21(70)$ & $19(90.5)$ & 0.098 \\
\hline Upper-Rectosigmoid & $6(27.3)$ & $6(18.3)$ & & $9(30)$ & $2(9.5)$ & \\
\hline \multicolumn{7}{|l|}{ Clinical T stage } \\
\hline $\mathrm{T} 2$ & $0(0)$ & $1(3)$ & 0.041 & $0(0)$ & $1(4.8)$ & 0.224 \\
\hline T3 & $13(56.5)$ & $8(24.2)$ & & $15(46.9)$ & $6(28.6)$ & \\
\hline $\mathrm{T} 4$ & $10(43.5)$ & $24(72.7)$ & & $17(53.1)$ & $14(66.7)$ & \\
\hline \multicolumn{7}{|l|}{ Clinical N stage } \\
\hline $\mathrm{N}-$ & $10(43.5)$ & $14(42.4)$ & 0.938 & $14(43.8)$ & $7(33.3)$ & 0.448 \\
\hline $\mathrm{N}+$ & $13(56.5)$ & $19(57.6)$ & & $18(56.3)$ & $14(66.7)$ & \\
\hline \multicolumn{7}{|l|}{ Pathological T stage } \\
\hline урто & $6(26.1)$ & $4(12.1)$ & 0.140 & $8(25)$ & $2(9.5)$ & 0.326 \\
\hline урт1-2 & $7(30.4)$ & $6(18.2)$ & & $8(25)$ & $5(23.8)$ & \\
\hline урT3-4 & $10(43.5)$ & $23(69.7)$ & & $16(50)$ & $14(66.7)$ & \\
\hline \multicolumn{7}{|l|}{ Pathological N stage } \\
\hline ypNO & $17(73.9)$ & $14(42.4)$ & 0.020 & $20(62.5)$ & $9(42.9)$ & 0.160 \\
\hline ypN1-2 & $6(26.1)$ & $19(57.6)$ & & $12(37.5)$ & $12(57.1)$ & \\
\hline \multicolumn{7}{|l|}{$\mathrm{pCR}$} \\
\hline Yes & $5(21.7)$ & $2(6.1)$ & 0.081 & $6(18.8)$ & $1(4.8)$ & 0.223 \\
\hline No & $18(78.3)$ & $31(93.9)$ & & $26(81.3)$ & $20(95.2)$ & \\
\hline \multicolumn{7}{|l|}{ Pathologic response } \\
\hline Good & $11(47.8)$ & $6(18.2)$ & 0.018 & $11(34.4)$ & $6(28.6)$ & 0.658 \\
\hline Poor & $12(52.2)$ & $27(81.8)$ & & $21(65.6)$ & $15(71.4)$ & \\
\hline \multicolumn{7}{|l|}{ LVI } \\
\hline No & $21(91.3)$ & $22(68.8)$ & 0.046 & $22(68.8)$ & $10(50)$ & 0.176 \\
\hline Yes & $2(8.7)$ & $10(31.3)$ & & $10(31.3)$ & $10(50)$ & \\
\hline \multicolumn{7}{|l|}{ PNI } \\
\hline No & $17(73.9)$ & $16(50)$ & 0.074 & $29(50.6)$ & $12(60)$ & 0.014 \\
\hline Yes & $6(26.1)$ & $16(50)$ & & $3(9.4)$ & $8(40)$ & \\
\hline \multicolumn{7}{|l|}{ Surgical margin } \\
\hline Negative & $19(82.6)$ & $29(90.6)$ & 0.379 & $29(50.6)$ & $16(80)$ & 0.275 \\
\hline Close/Positive & $4(17.4)$ & $3(9.4)$ & & $3(9.4)$ & $4(20)$ & \\
\hline
\end{tabular}

CRT: Chemoradiotherapy; NLR: Neutrophil-to-lymphocyte ratio; pCR: Pathological complete response; LVI: Lymphovascular invasion; PNI: Perineural invasion

and were included in the analysis. The median followup time was 45 months (3-98) with a median age of 63 years (range 30-83 years). All patients received threedimensional radiotherapy with 45 Gy in 25 fractions to the whole pelvis, 41 of whom received $5.4 \mathrm{~Gy}$ boost in 3 fractions to the primary tumor. Concurrent chemotherapy was applied to all patients except 4 patients. The most preferred chemotherapy regimen was 5-FU and it was used in 35 patients. Pathological evaluation after curative surgery revealed $12.5 \%$ pCR, $30.3 \%$ good pathological response and 58.9\% T-downstaging. At the last follow-up, 38 (67.9\%) patients were alive, 16 (28.5\%) had disease progression, 10 (17.8\%) had local recurrence, 11 (19.6\%) had distant metastasis.

The ROC analysis revealed favorable cut-off values of 2.87 (area under the curve [AUC]: 0.709, sen- 
sitivity $66 \%$, specificity $65 \%, \mathrm{p}=0.015$ ) for pre-CRT NLR associated with good pathological response and 8.68 (AUC: 0.678 , sensitivity $61 \%$, specificity $72 \%$, $\mathrm{p}=0.035$ ) for post-CRT NLR predicting survival, as shown in Figure 1a and b. According to these cut-off values, patients were stratified into two groups. A high pre-CRT NLR was significantly associated with clinical T4 stage $(\mathrm{p}=0.041)$, poor pathological response $(p=0.018)$ and LVI $(p=0.046)$. Most of pathological node positive patients were in the high pre-CRT NLR group significantly $(\mathrm{p}=0.020)$. The association between clinicopathological characteristics and NLR was detailed in Table 1.

Logistic regression analysis showed that patients in the low pre-CRT NLR group had better outcomes in terms of good pathological response compared to the high pre-CRT NLR group (OR 4.15, 95\% CI 1.23-13.76, $\mathrm{p}=0.021$ ) (Table 2). Clinical T4 stage was associated with poor pathological response (OR 3.63 [1.10-11.96], $\mathrm{p}=0.034)$. However, logistic regression analysis failed to show any parameters associated with pCR.

The 5-year OS was $65.7 \%$ for all patients. The 5-year OS in patients low and high pre-CRT NLR groups were $52.9 \%$ and $68.4 \%(\mathrm{p}=0.757)$. An elevated post-CRT NLR was a significantly poor prognostic factor for OS, with 5 -year OS rates of $74.4 \%$ and $46.6 \%$ for low and high post-CRT NLR groups ( $\mathrm{p}=0.006)$ (Fig. 2a). Clinical N stage $(\mathrm{p}=0.017)$, CEA $(\mathrm{p}=0.038)$, concurrent CRT $(\mathrm{p}<0.001)$ and radiation dose $(50.4 \mathrm{~Gy})(\mathrm{p}=0.007)$ were other significant factors affecting OS in the univariate analysis. post-CRT NLR ( $\mathrm{p}=0.023$ ), clinical $\mathrm{N}$ stage $(\mathrm{p}=0.024)$, CRT $(\mathrm{p}=0.003)$ and radiation dose $(50.4$ Gy) ( $\mathrm{p}=0.018$ ) were remained independent prognostic factors for OS in multivariate analysis (Table 3).

The 5-year DFS was $57.6 \%$ for all group. The 5-year DFS rates did not differ significantly between the low and high pre-CRT NLR groups (57.7\% vs. $58 \%$, $\mathrm{p}=0.860$ ). Low post-CRT NLR group showed a significantly improved 5-year DFS rate compared to high post-CRT NLR group (71.9\% vs. $35.3 \%, \mathrm{p}=0.018$ ) (Fig. $2 b)$. Clinical N stage $(p=0.010), P N I(p=0.008)$, surgical margin $(\mathrm{p}=0.039)$, CRT $(\mathrm{p}=0.001)$, radiation dose $(50.4$ Gy) ( $\mathrm{p}=0.046)$ and chemotherapy regimen $(\mathrm{p}=0.021)$ were other significant factors affecting DFS in the univariate analysis. post-CRT NLR $(\mathrm{p}=0.014)$, PNI $(\mathrm{p}=0.018)$, surgical margin $(\mathrm{p}=0.041)$, CRT $(\mathrm{p}=0.004)$ and chemotherapy regimen $(\mathrm{p}=0.038)$ were prognostic factors for DFS in multivariate analysis (Table 3 ).

The 5 -year LRRFS was $62.8 \%$ for all patients. The pre-CRT NLR was statistically insignificant for LR-

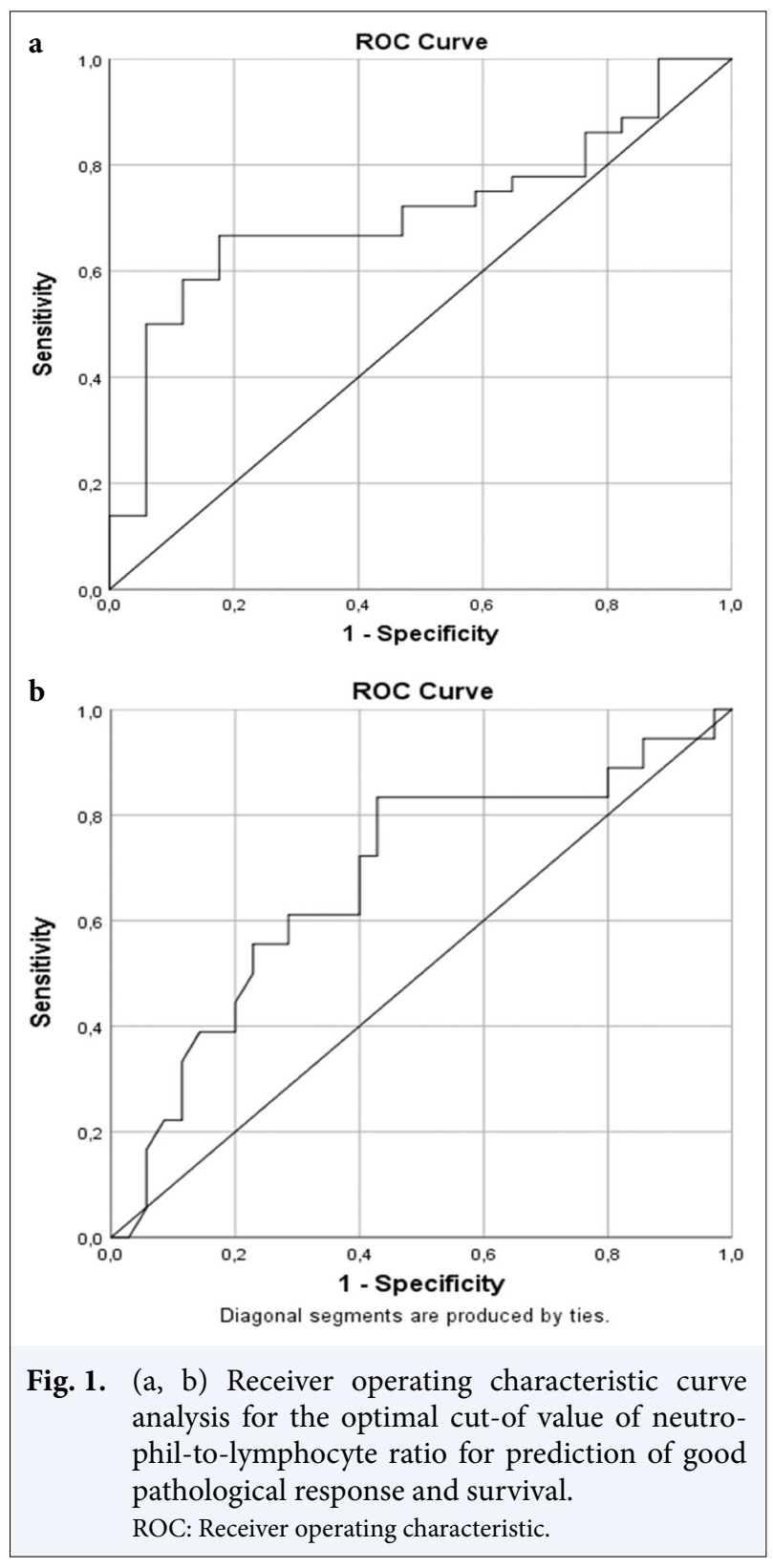

RFS, with 5-year LRRFS rates of $64.7 \%$ and $62.3 \%$ for low and high pre-CRT NLR groups $(\mathrm{p}=0.927)$. The 5 -year LRRFS rate was significantly better in low postCRT NLR group $(78.1 \%$ vs. $40.8 \%, \mathrm{p}=0.006)$ (Fig. 2c). Clinical N stage $(\mathrm{p}=0.005)$, PNI $(\mathrm{p}=0.029)$, CRT $(\mathrm{p}<0.001)$ and radiation dose (50.4 Gy) $(\mathrm{p}=0.007)$ were other significant factors affecting LRRFS in the univariate analysis. post-CRT NLR $(\mathrm{p}=0.019)$, clinical N stage $(\mathrm{p}=0.008)$, PNI $(\mathrm{p}=0.045)$, CRT $(\mathrm{p}=0.002)$ and radiation dose (50.4 Gy) $(\mathrm{p}=0.008)$ were remained prognostic factors for LRRFS in multivariate analysis (Table 3 ). 


\begin{tabular}{|c|c|c|c|c|}
\hline \multirow[t]{2}{*}{ Factors } & \multicolumn{2}{|c|}{$\begin{array}{l}\text { Good pathological } \\
\text { response }\end{array}$} & \multicolumn{2}{|c|}{ PCR } \\
\hline & OR (Cl \%95) & $\mathbf{p}$ & OR (Cl \%95) & $\mathbf{p}$ \\
\hline \multicolumn{5}{|l|}{ Clinical T Stage } \\
\hline T2-3 versus $T 4$ & $3.63(1.10-11.96)$ & 0.034 & $5.15(0.90-29.52)$ & 0.065 \\
\hline \multicolumn{5}{|l|}{ Clinical N Stage } \\
\hline $\mathrm{N}$ - versus $\mathrm{N}+$ & $1.17(0.23-5.84)$ & 0.845 & $1.06(0.13-8.64)$ & 0.956 \\
\hline \multicolumn{5}{|l|}{ Tumor diameter } \\
\hline$<3 \mathrm{~cm}$ versus $\geq 3 \mathrm{~cm}$ & $1.99(0.23-17.17)$ & 0.531 & $1.75(0.16-12.23)$ & 0.612 \\
\hline \multicolumn{5}{|l|}{ CEA } \\
\hline$<5$ versus $\geq 5$ & $0.68(0.07-6.25)$ & 0.740 & $1.26(0.74-4.57)$ & 0.413 \\
\hline \multicolumn{5}{|l|}{ Week } \\
\hline$<12$ versus $\geq 12$ & $0.81(0.14-4.50)$ & 0.818 & $0.28(0.04-1.77)$ & 0.177 \\
\hline \multicolumn{5}{|l|}{ Radiotherapy } \\
\hline CRT versus $R T$ & $0.29(0.20-4.36)$ & 0.376 & $1.93(0.07-51.64)$ & 0.695 \\
\hline \multicolumn{5}{|l|}{$\mathrm{RT}$ dose } \\
\hline 45 versus 50.4 & $1.92(0.22-16.35)$ & 0.558 & $3.19(0.19-53.48)$ & 0.420 \\
\hline \multicolumn{5}{|l|}{ Pre-CRT NLR } \\
\hline$<2.87$ versus $\geq 2.87$ & $4.15(1.23-13.76)$ & 0.021 & $2.74(0.37-20.15)$ & 0.320 \\
\hline
\end{tabular}

pCR: Pathological complete response; OR: Odds ratio; Cl: Confidence interval; CEA: Carcinoembryonic antigen; CRT: Chemoradiotherapy; RT: Radiotherapy; NLR: Neutrophil-to-lymphocyte ratio

\section{Discussion}

The current study has investigated the potential ability of NLR to serve as a prognostic marker for good pathological response and survival outcomes in LARC patients who were treated with neoadjuvant CRT. Our findings demonstrated that low pre-CRT NLR was an independent prognostic factor for good pathological response to neoadjuvant CRT, and low post-CRT NLR was associated with improved OS, DFS, LRRFS rates compared to high post-CRT NLR. However, no correlation was found between PCR and NLR.

The strengths of this study are as follows. First of all, the patients received treatment in a single center and their treatment was planned according to the same guidelines. Therefore, patient management did not differ between patients included in the study and biased results were avoided. Although the sample size was small, to obtain accurate results, we analyzed a highly selected proportion of patients undergoing surgical treatment with known pathology results. In addition, patients who did not have blood tests within the specified date ranges were excluded from the study in order to obtain the most reliable results. Finally, in order to make the study more deterministic, two different cutoff values that could be predictive of both good pathologic response to neoadjuvant CRT and survival were obtained separately from ROC analysis.
It is well known that the NLR is one of the most widely used indices as an indicator of immune inflammation, with its high prognostic ability to predict survival as it reflects host immune activity.[4,5] Previous studies have explored the prognostic utility of NLR in various solid tumors as well as colorectal tumors. $[7,8]$ Therefore, we have the knowledge that worse survival was observed in relation to higher NLR, confirmed by several published data. However, there is uncertainty about the effect of NLR on oncologic outcomes in patients undergoing neoadjuvant CRT for rectal cancer with known conflicting results. Dong et al.[9] performed a meta-analysis of seven studies including data from 959 patients treated with CRT/surgery or surgery, results demonstrated that high NLR was significantly related with worse OS, disease-free survival and recurrence-free survival. In contrast, a meta-analysis of 8 centers including 1237 patients compiled by Dudani et al.[10] failed to demonstrate the prognostic effect of NLR on survival. However, the following confounders should be noted when interpreting latter metaanalysis, which included only Canadian data, since the threshold value for NLR was not obtained for the patient population, the threshold value previously suggested by Templeton for various solid tumors was used instead of the value of their own data; [4] blood tests were taken over a wide period of time, 4 weeks before 


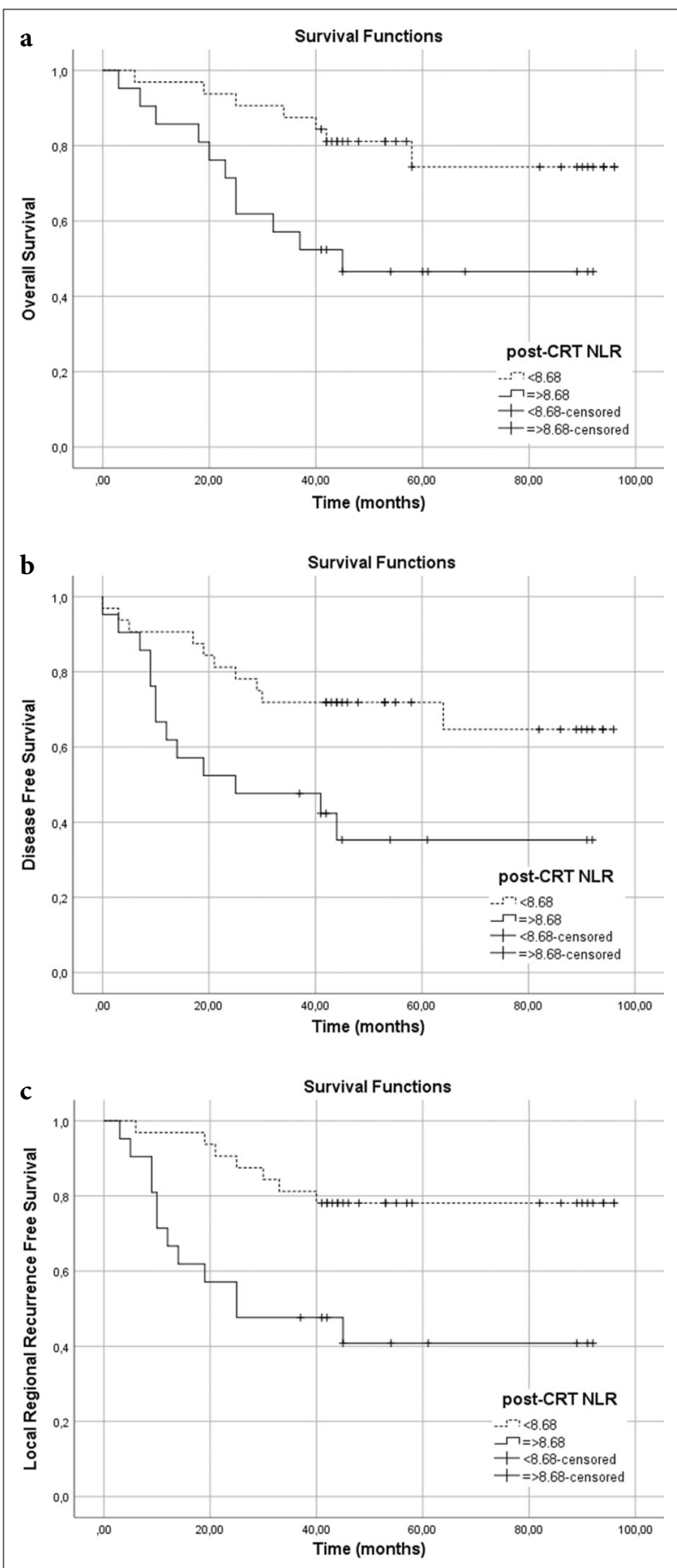

Fig. 2. (a) Kaplan-Meier graphs of Overall survival for patients according to post-chemoradiotherapy (CRT) neutrophil-to-lymphocyte ratio. (b) Kaplan-Meier graphs of disease free survival for patients according to post-CRT neutrophilto-lymphocyte ratio. (c) Kaplan-Meier graphs of local regioanal recurrence free survival for patients according to post-CRT neutrophil-tolymphocyte ratio. and 2 weeks after the start of CRT, were also accepted. It should be kept in mind that the indices may change, especially with the initiation of CRT. In our study, we evaluated baseline and post-treatment NLR, our results showed that post-CRT NLR was associated with OS, DFS, LRRFS rates, but pre-CRT NLR was not prognostic for survival by ROC analysis.

The link between systemic inflammation indices and tumor development has been demonstrated in many studies, but the primary goal was often to investigate the relationship between NLR and survival outcomes.[11,12] In particular, prediction of response assessment to neoadjuvant treatment according to NLR was beyond the scope. In the last decade, a limited number of studies examining this issue have been presented to the literature. [13-17] In this regard, the first data were obtained from the study presented by Krauthamer et al.[13] They reported that patients with low NLR had better complete response rates, but this relationship was only demonstrated in stage 3 patients. In another study that followed, NLR was found to be an independent predictive factor for tumor response and survival.[14] On the contrary, Shen et al.[15] reported no difference was found between the NLR groups in terms of tumor response. The reason why they could not obtain significant results may be that they could not determine the threshold value in the ROC analysis for their data and a cut-off value was selected from previous studies for analysis. In the other two studies, in which treatment response was evaluated with pre-and post-treatment NLR values, pre-treatment NLR was found to be predictive on tumor response, but this correlation with post-treatment NLR could not be demonstrated.[16,17] These two studies support our study with similar results.

According to previous data, increased $\mathrm{T}$ stage, lymph node-positive disease, presence of LVI, residual disease microscopically or macroscopically after surgery, poor pathological response to neoadjuvant therapy, are some of the unfavorable factors known to be prognostic for survival and recurrent disease.[18,19] Consistent with these data, we found that among these factors, high $\mathrm{T}$ stage, pathological node-positive disease, presence of LVI, and poor pathological response to neoadjuvant therapy were associated with the high pre-CRT NLR group. In addition, we found that increased T stage and high pre-CRT NLR were associated with a lower probability of achieving a good pathological response to neoadjuvant therapy. These findings may indicate that high NLR shares the same denominator with known poor prognostic factors. 


\begin{tabular}{|c|c|c|c|c|c|c|c|}
\hline \multirow{3}{*}{\multicolumn{2}{|c|}{ Variable }} & \multirow{2}{*}{\multicolumn{2}{|c|}{$\begin{array}{c}\text { OS } \\
\text { Univariate }\end{array}$}} & \multirow{2}{*}{\multicolumn{2}{|c|}{$\begin{array}{c}\text { DFS } \\
\text { Univariate }\end{array}$}} & \multirow{2}{*}{\multicolumn{2}{|c|}{$\begin{array}{c}\text { LRRFS } \\
\text { Univariate }\end{array}$}} \\
\hline & & & & & & & \\
\hline & & HR (\%95 Cl) & $\mathbf{p}$ & HR (\%95 Cl) & $\mathbf{p}$ & HR (\%95 Cl) & $\mathbf{P}$ \\
\hline Age & & & 0.357 & & 0.198 & & 0.364 \\
\hline \multicolumn{2}{|c|}{$<60$ versus $\geq 60$} & $1.62(0.57-4.56)$ & & $1.78(0.73-4.31)$ & & $1.55(0.59-4.07)$ & \\
\hline \multicolumn{2}{|c|}{ Gender } & & 0.198 & & 0.153 & & 0.081 \\
\hline \multicolumn{2}{|c|}{ Male versus female } & $0.54(0.21-1.37)$ & & $0.55(0.24-1.24)$ & & $0.45(0.18-1.10)$ & \\
\hline \multicolumn{2}{|c|}{ Tumor location } & & 0.456 & & 0.811 & & 0.648 \\
\hline \multicolumn{2}{|c|}{$\begin{array}{l}\text { Upper-rectosigmoid } \\
\text { versus low-mid }\end{array}$} & $1.48(0.52-4.15)$ & & $3.03(1.42-6.45)$ & & $1.26(0.46-3.48)$ & \\
\hline \multicolumn{2}{|c|}{ Clinical T stage } & & 0.082 & & 0.940 & & 0.134 \\
\hline \multicolumn{2}{|c|}{$\mathrm{T} 2-3$ versus $\mathrm{T} 4$} & $2.50(0.82-7.62)$ & & $1.03(0.45-2.36)$ & & $2.17(0.78-5.97)$ & \\
\hline \multicolumn{2}{|c|}{ Clinical N Stage } & & 0.017 & & 0.010 & & 0.005 \\
\hline \multicolumn{2}{|c|}{$\mathrm{N}$ - versus $\mathrm{N}+$} & $4.52(1.31-15.65)$ & & $3.68(1.37-9.90)$ & & $5.80(1.96-19.85)$ & \\
\hline \multicolumn{2}{|c|}{ Tumor Length } & & 0.314 & & 0.210 & & 0.418 \\
\hline \multicolumn{2}{|c|}{$<3 \mathrm{~cm}$ versus $\geq 3 \mathrm{~cm}$} & $0.54(0.16-1.78)$ & & $0.50(0.17-1.46)$ & & $0.63(0.21-1.89)$ & \\
\hline \multicolumn{2}{|c|}{ Pathologic T stage } & & 0.304 & & 0.457 & & 0.096 \\
\hline \multicolumn{2}{|c|}{ T1-3 versus $\mathrm{T} 4$} & $1.43(0.72-2.87)$ & & $1.25(0.69-2.28)$ & & $1.77(0.90-3.46)$ & \\
\hline \multicolumn{2}{|c|}{ Pathologic N stage } & & 0.094 & & 0.205 & & 0.205 \\
\hline \multicolumn{2}{|c|}{ No versus N1-2 } & $2.24(0.87-5.80)$ & & $1.68(0.75-2.76)$ & & $1.75(0.72-4.27)$ & \\
\hline \multicolumn{2}{|c|}{ Pathological Response } & & 0.758 & & 0.861 & & 0.927 \\
\hline \multirow{2}{*}{\multicolumn{2}{|c|}{ Good versus Poor }} & $0.85(0.32-2.28)$ & & $1.08(0.44-2.62)$ & & $1.04(0.40-2.72)$ & \\
\hline $\mathrm{pCR}$ & & & 0.742 & & 0.403 & & 0.616 \\
\hline Yes V & rsus no & $1.28(0.29-5.57)$ & & $1.85(0.43-7.90)$ & & $1.45(0.33-6.27)$ & \\
\hline Musineo & s Component & & 0.723 & & 0.547 & & 0.885 \\
\hline No v & sus yes & $0.81(0.26-2.48)$ & & $0.73(0.27-1.98)$ & & $1.07(0.39-2.96)$ & \\
\hline LVI & & & 0.365 & & 0.071 & & 0.273 \\
\hline No v & sus yes & $1.53(0.60-3.86)$ & & $2.10(0.93-4.71)$ & & $1.631(0.67-3.93)$ & \\
\hline PNI & & & 0.074 & & 0.008 & & 0.029 \\
\hline No v & sus yes & $2.45(0.91-6.57)$ & & $3.10(1.34-7.17)$ & & $2.79(1.11-7.09)$ & \\
\hline Surgical & Iargin & & 0.070 & & 0.039 & & 0.130 \\
\hline -vs C & se/+ & $2.81(0.91-8.58)$ & & $2.83(1.05-7.64)$ & & $2.34(0.77-7.02)$ & \\
\hline Radiothe & & & $<0.001$ & & 0.001 & & $<0.001$ \\
\hline CRT & arsus RT & $12.49(3.77-41.42)$ & & $6.72(2.19-20.61)$ & & $11.03(3.47-35.03)$ & \\
\hline RT dose & & & 0.007 & & 0.046 & & 0.007 \\
\hline 50.4 & ersus 45 & $0.27(0.11-0.70)$ & & $0.43(0.19-0.98)$ & & $0.30(0.12-0.72)$ & \\
\hline CT Regin & & & 0.206 & & 0.021 & & 0.122 \\
\hline 5-FU & ersus Cap & $1.99(0.683-5.80)$ & & $2.89(1.17-7.14)$ & & $2.22(0.81-5.97)$ & \\
\hline Post-CRT & & & 0.006 & & 0.018 & & 0.006 \\
\hline$<8.6$ & versus $\geq 8.68$ & $3.68(1.44-9.40)$ & & $2.72(1.18-6.26)$ & & $3.68(1.44-9.40)$ & \\
\hline Albumin & & & 0.824 & & 0.917 & & 0.940 \\
\hline$>3.5$ & ersus $\leq 3.5$ & $0.86(0.24-3.04)$ & & $0.94(0.31-2.79)$ & & $1.04(0.30-3.62)$ & \\
\hline $\mathrm{Hb}$ & & & 0.310 & & 0.245 & & 0.570 \\
\hline$>12$ & arsus $\leq 12$ & $0.59(0.21-1.62)$ & & $0.60(0.25-1.41)$ & & $0.74(0.30-1.93)$ & \\
\hline CEA & & & 0.038 & & 0.183 & & 0.086 \\
\hline$\leq 5 \mathrm{v}$ & sus $>5$ & $2.67(1.05-6.77)$ & & $1.73(0.77-3.52)$ & & $2.16(0.89-5.24)$ & \\
\hline Timing 0 & Surgery & & 0.334 & & 0.767 & & 0.253 \\
\hline$\geq 6 \mathrm{u}$ & eks versus $<6$ weeks & $0.61(0.23-1.63)$ & & $0.87(0.36-2.09)$ & & $0.58(0.23-1.46)$ & \\
\hline
\end{tabular}




\begin{tabular}{|c|c|c|c|c|c|c|}
\hline & \multicolumn{2}{|c|}{ Multivariate } & \multicolumn{2}{|c|}{ Multivariate } & \multicolumn{2}{|c|}{ Multivariate } \\
\hline & HR (\%95 Cl) & $\mathbf{p}$ & HR (\%95 Cl) & $\mathbf{p}$ & HR (\%95 Cl) & $\mathbf{p}$ \\
\hline Clinical N Stage & & 0.024 & & 0.024 & & 0.008 \\
\hline $\mathrm{N}$ - versus $\mathrm{N}+$ & $4.17(1.20-14.47)$ & & $3.16(1.16-8.58)$ & & $5.37(1.56-18.45)$ & \\
\hline $\mathrm{PNI}$ & & & & 0.018 & & 0.045 \\
\hline No versus yes & & & $3.09(1.21-7.89)$ & & $2.58(1.02-6.55)$ & \\
\hline Surgical margin & & & & 0.041 & & \\
\hline -vs close/+ & & & $3.06(1.08-8.31)$ & & & \\
\hline Radiotherapy & & 0.003 & & 0.004 & & 0.002 \\
\hline CRT versus $\mathrm{RT}$ & $7.73(1.9-29.91)$ & & $6.02(1.77-20.41)$ & & $8.38(2.18-32.20)$ & \\
\hline RT dose & & 0.018 & & 0.158 & & 0.008 \\
\hline 50.4 versus 45 & $0.29(0.11-0.81)$ & & $0.52(0.21-1.28)$ & & $0.21(0.08-0.82)$ & \\
\hline CT Regimen & & & & 0.038 & & \\
\hline 5-FU versus Cap & & & $3.08(1.06-8.95)$ & & & \\
\hline Post-CRT NLR & & 0.023 & & 0.014 & & 0.019 \\
\hline$<8.68$ versus $\geq 8.68$ & $3.11(1.17-8.28)$ & & $2.85(1.23-6.61)$ & & $3.68(1.24-10.92)$ & \\
\hline CEA & & 0.173 & & & & \\
\hline$\leq 5$ versus $>5$ & $1.95(0.74-5.14)$ & & & & & \\
\hline
\end{tabular}

OS: Overall survival; DFS: Disease free survival; LRRFS: Local regional recurrence free survival; HR: Hazard ratio; Cl: Confidence interval; pCR: Pathological complete response; LVI: Lymphovascular invasion; PNI: Perineural invasion; CRT: Chemoradiotherapy; RT: Radiotherapy; CT: Chemotherapy; 5-FU: 5-fluorouracil; Cap: Capesitabine; NLR: Neutrophil-to-lymphocyte ratio; Hb: Hemoglobin; CEA: Carcinoembryonic antigen

Nowadays, although it is not routinely recommended, it is included in the guidelines that surgical treatment may not be applied in some special cases with good response to therapy. Neoadjuvant therapy or total neoadjuvant therapy are the mainstays of this non-surgical approach. After clinicians evaluate the treatment response clinically with a detailed evaluation including rectal examination, rectal imaging, and endoscopic examinations instead of post-surgical pathological examination, "watch and wait" strategy can be applied in experienced centers in patients with clinically complete response.[20] However, clinical evaluation has some limitations due to the shortcomings of each of the above-mentioned methods due to difficulties in the post-treatment setting.[21] Since patients cannot be evaluated with full accuracy by clinical evaluation, it may result in downstaging of patients compared to pathological evaluation. In this context, systemic inflammation indices can contribute to post-treatment assessment regarding the creation of personalized treatment strategies, due to the prediction of response assessment and prognostic impact on survival.

\section{Limitations of the Study}

Some limitations of this study must be acknowledged, one of which is the small sample size obtained from a single-center study. This small sample size may also be responsible for our failure to demonstrate a significant relation between the well-known prognostic factors such as pCR and patient survival. Furthermore, there is the possibility of selection bias due to its retrospective design. Further analysis of a large population supported by a multicenter study is required to more accurately confirm the predictive value of NLR.

\section{Conclusion}

This study indicates that an elevated pre-CRT-NLR might be used as a poor pathological tumor response predictor in LARC patients treated with neoadjuvant CRT. In addition, low post-CRT NLR is associated with favorable OS, DFS, and LRRFS. Therefore, easily accessible and cost-effective NLR can be considered as a potential predictive marker to identify patients and establish personalized treatment strategies, which should be further warranted by prospective studies.

Peer-review: Externally peer-reviewed.

Conflict of Interest: All authors declared no conflict of interest.

Ethics Committee Approval: The study was approved by The University of Health Sciences, Samsun Training and Research Hospital Non-Interventional Clinical Research Ethics Committee (No: 2018/1/10, Date: 09/01/2018). 
Financial Support: This study has received no financial support.

Authorship contributions: Concept - E.D.S., Y.K., E.O.; Design - E.D.S., Y.K., N.Ş., Y.G.; Supervision - E.D.S, S.A.A.; Funding - None; Materials - E.D.S., Y.K., E.O., N.Ş., B.K.Y., İ.M.Y.; Data collection and/or processing - E.D.S., E.O., N.S., B.K.Y., İ.M.Y.; Data analysis and/or interpretation - E.D.S., B.K.Y., I.M.Y., S.A.A., Y.G.; Literature search - E.D.S., E.O., N.Ş., B.K.Y., İ.M.Y.; Writing - E.D.S., S.A.A.; Critical review - E.D.S., S.A.A., Y.G.

\section{References}

1. Sauer R, Becker H, Hohenberger W, Rödel C, Wittekind C, Fietkau R, et al. German Rectal Study Group. Preoperative versus postoperative chemoradiotherapy for rectal cancer. N Engl J Med 2004;351(17):1731-40.

2. Maas M, Nelemans PJ, Valentini V, Das P, Rödel C, Kuo LJ, et al. Long-term outcome in patients with a pathological complete response after chemoradiation for rectal cancer: a pooled analysis of individual patient data. Lancet Oncol 2010;11(9):835-44.

3. Grivennikov SI, Greten FR, Karin M. Immunity, inflammation, and cancer. Cell 2010;140(6):883-99.

4. Templeton AJ, McNamara MG, Šeruga B, Vera-Badillo FE, Aneja P, Ocaña A, et al. Prognostic role of neutrophil-to-lymphocyte ratio in solid tumors: a systematic review and meta-analysis. J Natl Cancer Inst 2014;106(6):dju124.

5. Guthrie GJ, Charles KA, Roxburgh CS, Horgan PG, McMillan DC, Clarke SJ. The systemic inflammation based neutrophil-lymphocyte ratio: experience in patients with cancer. Crit Rev Oncol Hematol 2013;88(1):218-30.

6. Ryan R, Gibbons D, Hyland JM, Treanor D, White A, Mulcahy HE, et al. Pathological response following long-course neoadjuvant chemoradiotherapy for locally advanced rectal cancer. Histopathology 2005;47(2):141-6.

7. Hung HY, Chen JS, Yeh CY, Changchien CR, Tang $\mathrm{R}$, Hsieh PS, et al. Effect of preoperative neutrophillymphocyte ratio on the surgical outcomes of stage II colon cancer patients who do not receive adjuvant chemotherapy. Int J Colorectal Dis 2011;26(8):105965.

8. Ishizuka $M$, Nagata $\mathrm{H}$, Takagi $\mathrm{K}$, Iwasaki $\mathrm{Y}$, Kubota $\mathrm{K}$. Combination of platelet count and neutrophil to lymphocyte ratio is a useful predictor of postoperative survival in patients with colorectal cancer. $\mathrm{Br} \mathrm{J}$ Cancer 2013;109(2):401-7.

9. Dong YW, Shi YQ, He LW, Su PZ. Prognostic significance of neutrophil-to-lymphocyte ratio in rectal cancer: a meta-analysis. Onco Targets Ther 2016;9:3127-34.
10. Dudani S, Marginean H, Tang PA, Monzon JG, Raissouni S, Asmis TR, et al. Neutrophil-to-lymphocyte and platelet-tolymphocyte ratios as predictive and prognostic markers in patients with locally advanced rectal cancer treated with neoadjuvant chemoradiation. BMC Cancer 2019;19:664.

11. Carruthers R, Tho LM, Brown J, Kakumanu S, McCartney E, McDonald AC. Systemic inflammatory response is a predictor of outcome in patients undergoing preoperative chemoradiation for locally advanced rectal cancer. Colorectal Dis 2012;14(10):e701-7.

12. Nagasaki T, Akiyoshi T, Fujimoto Y, Konishi T, Nagayama S, Fukunaga Y, et al. Prognostic impact of neutrophil-to-lymphocyte ratio in patients with advanced low rectal cancer treated with preoperative chemoradiotherapy. Dig Surg 2015;32(6):496-503.

13. Krauthamer M, Rouvinov K, Ariad S, Man S, Walfish $\mathrm{S}$, Pinsk I, et al. A study of inflammation-based predictors of tumor response to neoadjuvant chemoradiotherapy for locally advanced rectal cancer. Oncology 2013;85(1):27-32.

14. Kim TG, Park W, Kim H, Choi DH, Park HC, Kim $\mathrm{SH}$, et al. Baseline neutrophil-lymphocyte ratio and platelet-lymphocyte ratio in rectal cancer patients following neoadjuvant chemoradiotherapy. Tumori 2019;105(5):434-40.

15. Shen J, Zhu Y, Wu W, Zhang L, Ju H, Fan Y, et al. Prognostic role of neutrophil-to-lymphocyte ratio in locally advanced rectal cancer treated with neoadjuvant chemoradiotherapy. Med Sci Monit 2017;23:315-24.

16. Kim IY, You SH, Kim YW. Neutrophil-lymphocyte ratio predicts pathologic tumor response and survival after preoperative chemoradiation for rectal cancer. BMC Surg 2014;14:94.

17. Lee IH, Hwang S, Lee SJ, Kang BW, Baek D, Kim HJ, et al. Systemic inflammatory response after preoperative chemoradiotherapy can affect oncologic outcomes in locally advanced rectal cancer. Anticancer Res 2017;37(3):1459-65.

18. Rödel C, Graeven U, Fietkau R, Hoenberger W, Hothorn T, Arnold D, et al. Oxaliplatin added to fluorouracil-based preoperative chemoradiotherapy and postoperative chemotherapy of locally advanced rectal cancer (the German CAO/ARO/AIO-04 study): final results of the multicentre, open-label, randomised, phase 3 trial. Lancet Oncol 2015;16(8):979-89.

19. Theodoropoulos G, Wise WE, Padmanabhan A, Kerner BA, Taylor CW, Aguilar PS, et al. T-level downstaging and complete pathologic response after preoperative chemoradiation for advanced rectal cancer result in decreased recurrence and improved diseasefree survival. Dis Colon Rectum 2002;45(7):895-903.

20.van der Valk MJM, Hilling DE, Bastiaannet E, Kranenbarg EMK, Beets GL, Figueiredo NL, et al. Long 
term outcomes of clinical complete responders after neoadjuvant treatment for rectal cancer in the International Watch \& Wait Database (IWWD): An international multicentre registry study. Lancet 2018;391(10139):2537-45.
21. Andras D, Crisan D, Craciun R, Nemes A, Caziuc A, Drasovean $\mathrm{R}$, et al. Neutrophil-to-lymphocyte ratio: a hidden gem in predicting neoadjuvant treatment response in locally advanced rectal cancer? J BUON 2020;25(3):1436-42. 